Article

\title{
Desaturase Activity and the Risk of Type 2 Diabetes and Coronary Artery Disease: A Mendelian Randomization Study
}

\author{
Susanne Jäger ${ }^{1,2}\left({ }^{\circ}\right.$, Rafael Cuadrat ${ }^{1,2} \oplus$, Per Hoffmann ${ }^{3,4} \oplus$, Clemens Wittenbecher ${ }^{1,2,5}$ \\ and Matthias B. Schulze ${ }^{1,2,6, *(D)}$ \\ 1 Department of Molecular Epidemiology, German Institute of Human Nutrition Potsdam-Rehbruecke, \\ 14558 Nuthetal, Germany; susanne.jaeger@dife.de (S.J.); Rafael.Cuadrat@dife.de (R.C.); \\ cwittenbecher@hsph.harvard.edu (C.W.) \\ 2 German Center for Diabetes Research (DZD), 85764 Neuherberg, Germany \\ 3 Human Genomics Research Group, Department of Biomedicine, University of Basel, 4031 Basel, Switzerland; \\ per.hoffmann@unibas.ch \\ 4 Institute of Human Genetics, University of Bonn, School of Medicine \& University Hospital Bonn, \\ 53105 Bonn, Germany \\ 5 Department of Nutrition, Harvard T.H. Chan School of Public Health, Boston, MA 02115, USA \\ 6 Institute of Nutritional Science, University of Potsdam, 14558 Nuthetal, Germany \\ * Correspondence: mschulze@dife.de; Tel.: +49-33200-882434
}

Received: 10 June 2020; Accepted: 25 July 2020; Published: 28 July 2020 updates

\begin{abstract}
Estimated $\Delta 5$-desaturase (D5D) and $\Delta 6$-desaturase (D6D) are key enzymes in metabolism of polyunsaturated fatty acids (PUFA) and have been associated with cardiometabolic risk; however, causality needs to be clarified. We applied two-sample Mendelian randomization (MR) approach using a representative sub-cohort of the European Prospective Investigation into Cancer and Nutrition (EPIC)-Potsdam Study and public data from DIAbetes Genetics Replication And Meta-analysis (DIAGRAM) and Coronary ARtery DIsease Genome wide Replication and Meta-analysis (CARDIoGRAM) genome-wide association studies (GWAS). Furthermore, we addressed confounding by linkage disequilibrium (LD) as all instruments from FADS1 (encoding D5D) are in LD with FADS2 (encoding D6D) variants. Our univariable MRs revealed risk-increasing total effects of both, D6D and D5D on type 2 diabetes (T2DM) risk; and risk-increasing total effect of D6D on risk of coronary artery disease (CAD). The multivariable MR approach could not unambiguously allocate a direct causal effect to either of the individual desaturases. Our results suggest that D6D is causally linked to cardiometabolic risk, which is likely due to downstream production of fatty acids and products resulting from high D6D activity. For D5D, we found indication for causal effects on T2DM and CAD, which could, however, still be confounded by LD.
\end{abstract}

Keywords: $\Delta 5$-desaturase; $\Delta 6$-desaturase; type 2 diabetes; coronary artery disease; Mendelian randomization; multivariable Mendelian randomization; FADS-gene-cluster; fatty acids

\section{Introduction}

$\Delta 5$-Desaturase (D5D) and $\Delta 6$-desaturase (D6D) are key enzymes for the synthesis of longer chain poly-unsaturated fatty acids (PUFA) from plant-derived precursor fatty acids linoleic acid (LA) and $\alpha$-linolenic acid (ALA) (see Figure 1a). 

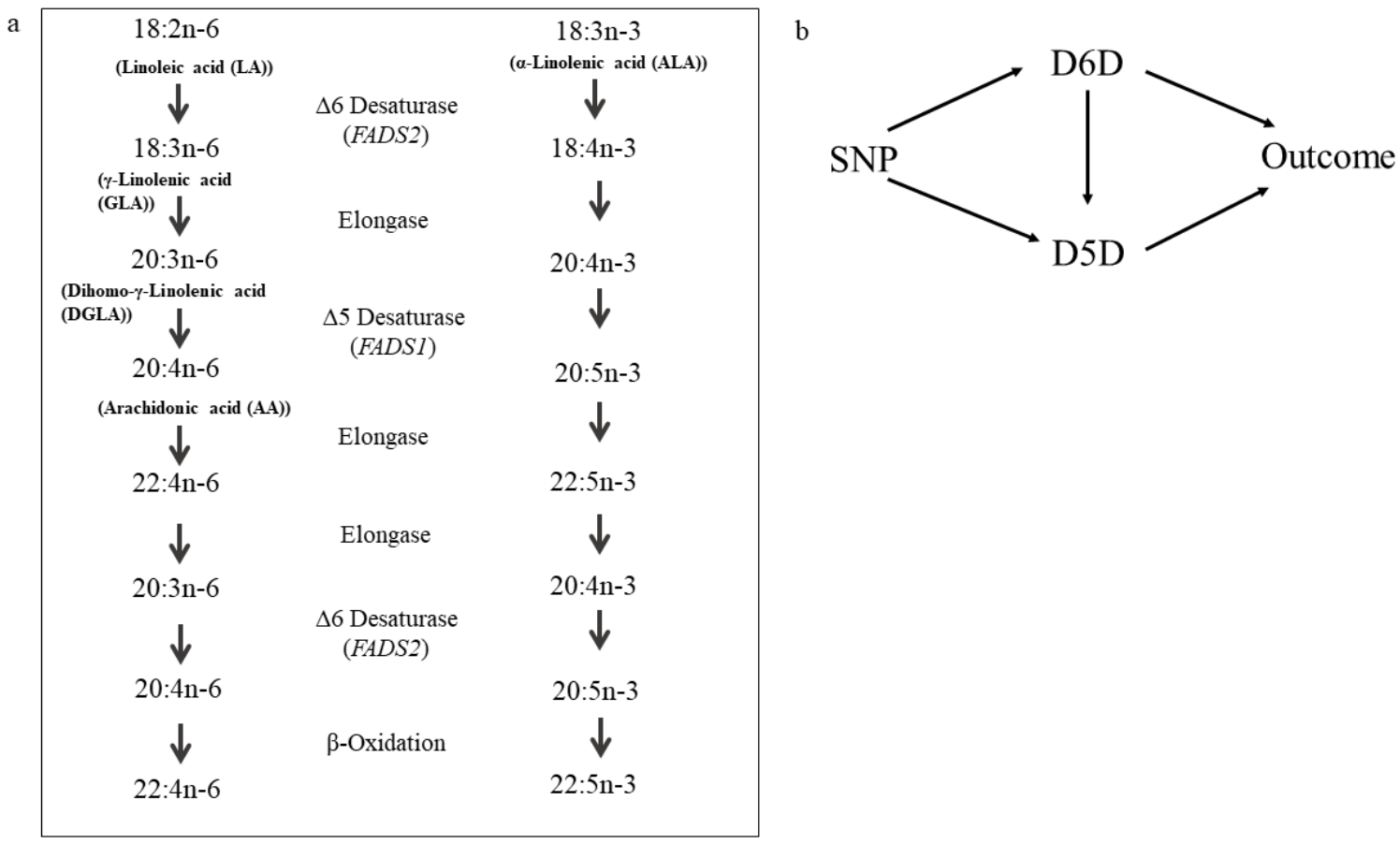

Figure 1. (a) Conversion of linoleic acid and $\alpha$-linolenic acid to longer-chain n-6- and n-3 polyunsaturated fatty acids by the action of $\Delta 6$-desaturase, $\Delta 5$-desaturase and elongases. Adapted from Kröger et al. [1]. (b) Hypothesized relationship between genetic variants, $\Delta 6$-desaturase (D6D), $\Delta 5$-Desaturase (D5D) and the investigated outcomes type 2 diabetes and coronary artery disease. The arrow from D6D to D5D relies on the biologically underlying mechanisms as depicted in panel a; however, methodologically in the multivariable MR the arrow could be in any direction. Confounders are omitted from this figure for clarity. Pleiotropic effects by pathways other than D6D or D5D are not considered in this multivariable MR.

Prospective cohort studies used product-to-precursor ratios of fatty acids measured in blood fractions, representing surrogate markers of estimated liver desaturase activity [1]. Overall, study findings support that a higher estimated D6D activity (ratio of 18:3n-6/18:2n-6) is related to an increased type 2 diabetes (T2DM) risk, while the contrary is the case for estimated D5D activity (20:4n-6/20:3n-6) [1-3]. Furthermore, higher D6D activity is linked to higher cardiovascular mortality [4] and higher estimated D5D activity to lower cardiovascular mortality and coronary heart disease (CHD) risk $[4,5]$.

To investigate causal relationships between risk factors and disease outcomes, genetic variants can be used as instrumental variables in Mendelian randomization (MR). The principle is based on Mendels's second law of inheritance, stating that the assignment of alleles is random during meiosis [6].

In humans, D5D and D6D are encoded by FADS1 and FADS2 genes [7] and SNPs in that gene region have been reliably linked to desaturase activities $[1,8,9]$ and cardiometabolic traits $[10,11]$. Recent MR studies have linked single fatty acids to T2DM risk [12] and CVD risk [13]. However, they are limited by the high correlation between the single fatty acids and hence individual effects could largely not be elucidated. A previous MR study found a direct relation for D6D activity and tended to support an inverse relation for D5D activity using a FADS1-SNP as genetic instrument [14]. However, the FADS-gene cluster is characterized by high linkage disequilibrium (LD) where all instruments from FADS1 are in LD with FADS2 variants [1,7]. Therefore, it is hard to select suitable genetic instruments for MR to disentangle the causal nature of each desaturase on the respective outcome without the limitation of confounding by LD. Furthermore, genetic instruments might associate with multiple exposures due to pleiotropic or mediating effects or both [15]. By applying multivariable $\mathrm{MR}$, we are able to use multiple genetic instruments to estimate direct effects of both desaturases 
simultaneously in one model [15], providing novel insights into causal inference of desaturase activity and cardiometabolic outcomes (see Figure 1b).

In contrast to previous MR studies on PUFA metabolism, we aim to estimate the causal effects of D6D and D5D activity, instead of single fatty acids, on the risk of T2DM and CAD. To this end, we selected suitable genetic instruments in genome-wide association analyses on estimated D6D and D5D activities. Disease associations were drawn from large public genome-wide association studies (GWAS) on T2DM and coronary artery disease (CAD). In univariable and multivariable two-sample MR studies we estimated the causal effects of D6D and D5D activity on T2DM risk and CAD risk, accounting for interrelations between single desaturases and hence single fatty acids. Finally, we address the phenomenon of confounding by LD within the FADS gene cluster, which was not or not sufficiently accounted for in previous MR studies.

\section{Materials and Methods}

\subsection{Study Population}

\subsubsection{Individual-Level Data from EPIC-Potsdam}

The European Prospective Investigation into Cancer and Nutrition (EPIC)-Potsdam study consists of 27,548 participants recruited between 1994 and 1998 from the general population in Potsdam and surroundings [16]. We used a random sample within the EPIC-Potsdam study, described in detail previously [17]. Briefly, a sub-cohort of 2500 individuals was randomly selected from 26,444 participants who provided blood samples at baseline. Participants with prevalent diabetes, diabetes medication at baseline or prevalent myocardial infarction were excluded. Further exclusion criteria were missing genetic data and missing or implausible data on fatty acid measurements, leaving 1853 individuals for analyses in the sub-cohort (Supplemental Figure S1).

All participants provided written informed consent. The study was approved by the ethics committee of the State of Brandenburg, Germany. All procedures were in accordance with the ethical standards of the institutional and/or national research committee and with the 1964 Helsinki declaration and its later amendments or comparable ethical standards.

\subsubsection{Summary-Level Data from DIAGRAM and CARDIoGRAM}

For outcome associations, we used summary-level data obtained from the DIAbetes Genetics Replication And Meta-analysis (DIAGRAM) consortium without BMI adjustment [18] and from meta-analysis of UK Biobank SOFT CAD GWAS with Coronary ARtery DIsease Genome wide Replication and Meta-analysis (CARDIoGRAM) plus The Coronary Artery Disease (C4D) Genetics (CARDIoGRAMplusC4D) 1000 Genomes-based GWAS and the Myocardial Infarction Genetics and CARDIoGRAM Exome [19]. The SOFT CAD phenotype incorporates self-reported angina or other evidence of chronic coronary heart disease [19].

\subsection{DNA-Extraction, Genotyping and Quality Control}

The DNA was extracted from buffy coats using the chemagic DNA Buffy Coat Kit on a Chemagic Magnetic Separation Module I (PerkinElmer chemagen Technologies, Baesweiler, Germany) according to the manufacturer's manual. Samples from EPIC-Potsdam participants were genotyped with three different genotyping arrays: Human660W-Quad_v1_A $(n=355)$, HumanCoreExome-12v1-0_B $(n=622)$ and Illumina Infinium OmniExpressExome-8v1-3_A DNA Analysis BeadChip $(n=1349)$. Genotyping and quality control of the Human660W-Quad_v1_A and HumanCoreExome-12v1-0_B chips was described elsewhere [20]. Genotyping using the Illumina Infinium OmniExpressExome-8v1-3_A DNA Analysis BeadChip was performed in the Life and Brain Center in Bonn, Germany. The DNA was processed according to the manufacturer's instruction using an automatized, LIMS controlled workflow and the arrays were finally scanned using an Illumina iScan bead arrays reader. Genotype calling 
and quality control of the samples were carried out jointly in all 1349 participants using Illumina's GenomeStudio v2011.1 software suite. For rare variants, zCall (threshold $=7$ ) was applied [21]. Exclusions were due to low call rate, discordant sex information, related or duplicated individuals and divergent ancestry (deviating from CEU) [22-24], leaving a sample size of 1292 participants genotyped with the Illumina Infinium OmniExpressExome-8v1-3_A DNA Analysis BeadChip. Overall, we had data for $n=2269$ samples available. Phasing and imputation were conducted using the Michigan Imputation Service [25]. The Haplotype Reference Consortium (release 1.1) served as reference panel [26]. Pre-phasing was applied using Eagle2 [27]. Imputation was carried out in four separate datasets (one for each chip or two for the HumanCoreExome-12v1-0_B chip) using minimac3 [25]. Imputed files were merged using bcftools [28], keeping the minimal R2 score. Afterwards, SNPs were filtered by R2 keeping those with values bigger than 0.6. Pre- and post-imputation tools (HRC-1000G-check-bim.v4.2.9, icv.1.0.5) for checking data quality were applied [29].

\subsection{Determination of Desaturase Activities}

Thirty milliliters of blood were obtained from EPIC-Potsdam participants during baseline examination. Plasma, serum, erythrocytes and buffy coat were stored at $-80{ }^{\circ} \mathrm{C}$. The erythrocyte membrane fatty acids were analyzed between February and June 2008. Thirty-two fatty acids were determined by gas chromatography and expressed as the percentage of total fatty acids present in the chromatogram [14]. Estimated D5D activity was determined as the ratio arachidonic acid (AA)/dihomo- $\gamma$-linolenic acid (DGLA) (20:4n-6/20:3n-6), and D6D activity as the ratio $\gamma$-linolenic acid (GLA)/linoleic acid (LA) (18:3n-6/18:2n-6), as done previously [1].

\subsection{Statistical Analysis}

We used the Statistical Analysis System (SAS) Enterprise Guide 7.1 with SAS version 9.4 (SAS Institute Inc., Cary, NC, USA) for data management and data preparation. For data filtering we used QCtool v1.4 and for GWAS we used SNPtest v2.5.2 [30]. We performed Mendelian Randomization analyses with R (version 3.5.2 (2018-12-20)) using the TwoSampleMR (v0.4.26) [31], the Mendelian Randomization (v0.4.1 and v0.4.2) [32], Radial MR (0.4) [33] and MVMR (0.2) [34] R packages. To visualize LD of the FADS-gene region and genetic instruments we utilized Plink v1.07, plink v1.90 [35] and Haploview v4.1 [36].

\subsubsection{Selecting Genetic Instruments in Genome-Wide Association Study}

SNPs were filtered by SNP missing-rate (removed $\geq 0.05$ ), minor allele frequency (MAF) (removed out of interval [0.05-0.5]) and Hardy-Weinberg equilibrium (removed -log10( $p$-value) $\geq 3$ ). Genetic instruments were obtained from GWAS on natural log-transformed and standardized (mean $=0$; $\mathrm{SD}=1$ ) estimated D5D and D6D using $\mathrm{n} \sim 5,340,003$ markers as exposures. We considered a $p$-value as genome-wide significant at $p<9.36 \times 10^{-9}[0.05 / 5,340,003]$. Suggestive significance threshold was defined as $p<1.00 \times 10^{-5}$. We assumed an additive genetic model, adjusted for age at recruitment and sex. Variants were mapped to Ensembl annotation version 87 (GRCh37) [37] and we used the Ensembl Variant Effect Predictor for annotation [38].

\subsubsection{Mendelian Randomization}

We conducted univariable two-sample MR studies using single desaturases as exposures on cardiometabolic outcomes. Effect estimates of the association between genetic instruments and desaturases were obtained from EPIC-Potsdam data and effect estimates of the SNP-T2DM and SNP-CAD associations were used from public summary GWAS data on T2DM [18] and CAD [19]. We selected gene-wide significant $(p<[0.05 / 169])$ instrumental variables and performed clumping according to LD. Therefore, SNPs within a window of 10,000 kb and being in LD as defined by $R^{2} \geq 0.3$ were removed (Figure 2). The SNP with the lowest $p$-value was retained. Within the MR analysis we accounted for their correlation among each other estimated in 502 European samples 
from 1000 Genomes phase 3 [39]. We repeated the analysis by additionally including independent genome-wide significant hits $\left(p<9.36 \times 10^{-9}\right)$ that were not located within the FADS gene region (with a clumping-threshold of 0.001$)$.

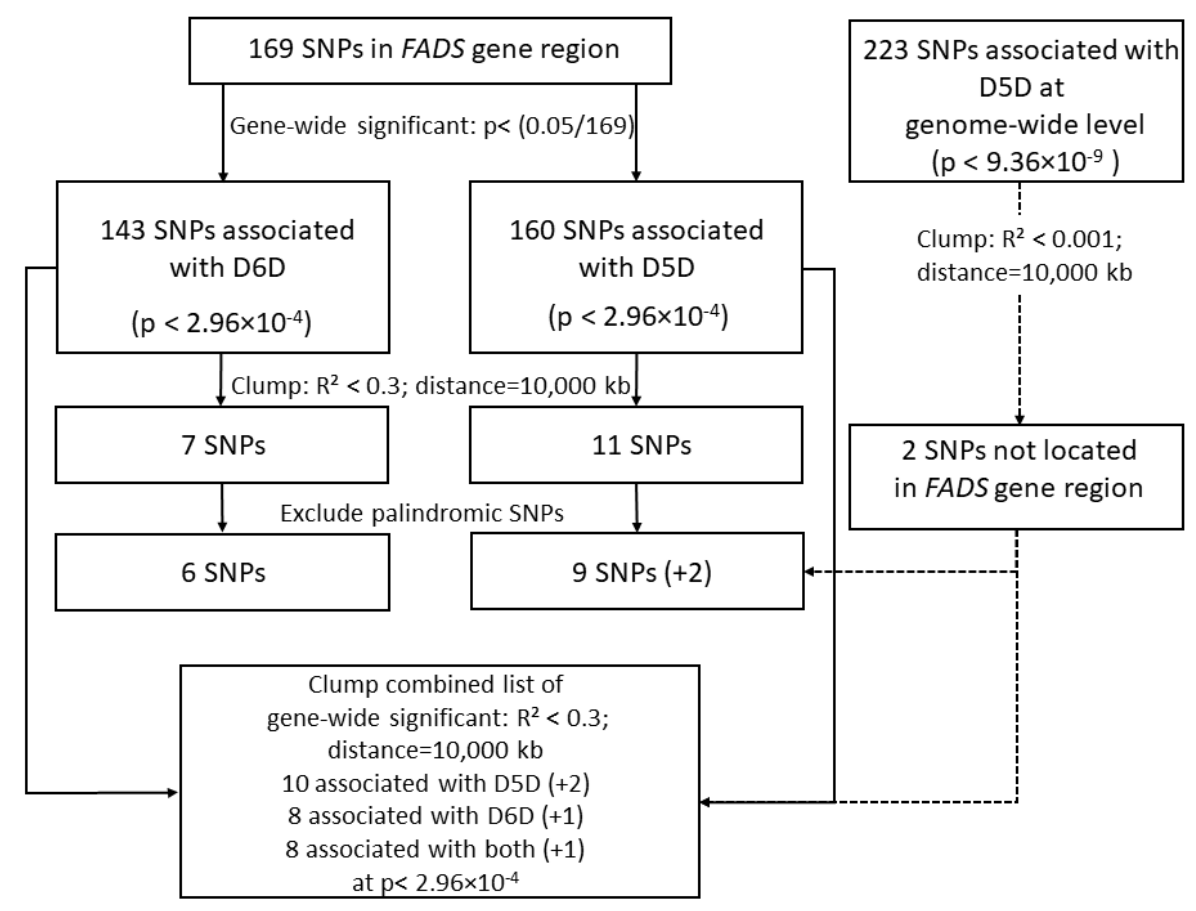

Figure 2. Flow chart of inclusion and exclusion of SNPs into the analysis. First, we restricted to the set of SNPs that were located within the FADS gene region (Chr 11: 61560452-61659523). Next, we clumped the GWAS results separately for D6D and D5D to identify independent SNPs for both traits for univariable MR approach. Finally, we clumped a combined list of SNPs for both traits for multivariable MR. This resulted in ten SNPs for D5D and eight SNPs for D6D at gene-wide significance level $p<2.96 \times 10^{-4}$. There were eight SNPs that associated with both desaturases. All SNPs were included in the analysis. Additionally, two SNPs at genome-wide significance level for D5D were included.

Data were harmonized for the direction of effects between exposure and outcome associations and palindromic SNPs were excluded. We used an inverse variance weighted (IVW) meta-analysis of SNP specific Wald ratios (SNP-outcome estimate divided by SNP-exposure estimate) using random effects, to obtain causal estimates for the desaturase activities on T2DM or CAD risk. MR-Egger method [40] was used in sensitivity analyses. Heterogeneity was assessed by the Cochran's Q statistic and we performed RadialMR [33] to identify outliers with the largest contribution to the $Q$ statistic. Outlying genetic instruments were removed and the data were re-analyzed. Radial MR analysis was conducted using second order weights and an $\alpha$ level of 0.05 or 0.1 .

We applied a multivariable MR approach [15] combining both desaturases (see Figure 1b). First, we selected all gene-wide significant instruments for each estimated desaturase activity. This combined list was again pruned with $R^{2}<0.3$ leaving ten independent instruments. We obtained the SNP effects on the other desaturase activity and vice versa. Extraction of the outcome GWAS results and data harmonization was conducted as described for the univariable MR. We applied the IVW multivariable MR performing multivariable weighted linear regression with the intercept term set to zero and accounting for the correlation structure among the ten FADS variants. We additionally included genome-wide significant hits $\left(p<9.36 \times 10^{-9}\right)$ that were not located within the FADS gene region. As sensitivity analyses, we used multivariable MR-Egger for two-sample summary data [41]. We calculated F-statistics to evaluate the presence of weak instruments within the multivariable MR analysis and adjusted for those by minimizing the Q-statistic allowing for heterogeneity using 
"qhet_mvmr" function with 1000 bootstrap iterations from the MVMR package [34]. Phenotypic correlation was obtained from EPIC-Potsdam data. To account for horizontal pleiotropic effects by other pathways than considered in the multivariable approach, we used Causal Analysis Using Summary Effect estimates (CAUSE) [42].

\subsubsection{Investigation of LD within the FADS-Gene Cluster}

To exclude possibly confounding by LD, we restricted the MR analysis for D6D on variants that are located in FADS2 only and are not in LD $\left(R^{2}<0.45\right)$ with SNPs located in FADS1-FADS2 LD Block. The selection was based on a LD plot of all 143 D6D GWAS Hits available within the FADS-gene region in EPIC-Potsdam. For D5D, we included only genome-wide hits outside from FADS due to the strong LD of FADS1 variants with variants in FADS2.

\section{Results}

\subsection{Selection of Genetic Instruments}

GWAS were conducted in EPIC-Potsdam with 1853 participants. Baseline characteristics are illustrated in Table 1.

Table 1. Baseline characteristics, EPIC-Potsdam random sample.

\begin{tabular}{cc}
\hline & EPIC-Potsdam \\
\hline $\mathrm{N}$ & 1853 \\
\hline Sex (\% men) & 37.1 \\
\hline $\begin{array}{c}\text { Age in years; median (interquartile range) } \\
\text { Waist circumference in cm; mean (SD) } \\
\text { median (interquartile range) }\end{array}$ & $49.0(15.5)$ \\
\hline $\begin{array}{c}\Delta 5 \text {-desaturase activity (20:4n-6/20:3n-6); } \\
\text { mean (SD) }\end{array}$ & $85.3(12.6)$ \\
\hline Lipid medication (\%) & $0.005(0.003)$ \\
\hline SD, standard deviation.
\end{tabular}

Genetic variants within the FADS gene region were strongly associated with estimated D6D (Supplemental Table S1, Supplemental Figure S2) and D5D activity (Supplemental Table S2, Supplemental Figure S2). For D6D, we identified 143 FADS-gene-wide significant $(p<$ [0.05/169]) variants of which after LD clumping seven independent variants $\left(R^{2}<0.3\right)$ remained (Supplemental Table S1, Supplemental Figure S3). All of them were available within DIAGRAM and one (rs174607) could not be found within CAD outcome data. However, this SNP (rs174607) was labeled as palindromic variant and was therefore excluded also from the diabetes analysis (Supplemental Table S2).

Regarding D5D, 160 SNPs showed FADS-gene-wide significance ( $p<$ [0.05/169]) resulting in 11 independent variants $\left(R^{2}<0.3\right)$ (Supplemental Table S2, Supplemental Figure S3). All of them were available within DIAGRAM and one (rs174607) was not available for CAD outcome data. Two variants (rs174607, rs174565) were excluded from the diabetes analysis because of being palindromic and are therefore not presented in Supplemental Table S2.

Furthermore, our GWAS identified two novel loci associated with D5D activity at genome-wide significance level: an intronic variant (rs2608073) at chromosome 3 within the RP11-372E1.4 locus and an intronic variant (rs11644601) at the RRN3; PDXDC1 locus at chromosome 16 (Supplemental Table S2 and Supplemental Figure S2). 


\subsection{Mendelian Randomization}

\subsubsection{Univariable Mendelian Randomization}

Causal Estimates for Desaturase Activities on Risk of Type 2 Diabetes

The IVW estimate indicated a positive total effect of D6D (odds ratio (OR) [95\% confidence interval $(\mathrm{CI})]=1.08$ [1.06-1.09]) on T2DM (Table 2, Supplemental Figure S4). The results showed no indication of heterogeneity between the SNPs (Cochran's $Q$ value $=7.17, p=0.21$ ). The MR-Egger regression showed a positive intercept $0.012(\mathrm{SE}=0.009) p=0.185)$ and OR (1.04 [0.98-1.10]) (Supplemental Table S3), not supporting directional horizontal pleiotropy with regards to T2DM.

For D5D, we found a positive total effect (OR = 1.03 [1.01-1.04]) on T2DM (Table 2, Supplemental Figure S5). There was evidence for heterogeneity among the SNPs (Cochran's Q value $=28.25$, $p=0.0004)$. The MR-Egger regression showed a positive intercept $(0.011(\mathrm{SE}=0.011), p=0.306)$ and no association (OR = 1.00 [0.98-1.05]) (Supplemental Table S3), not indicating presence of directional horizontal pleiotropy. Based on the Radial MR, we identified two outlying SNPs (rs174602, rs508768) for the D5D-T2DM analysis (Supplemental Figure S6). After outlier removal, inverse variance weighted and MR-Egger effect estimates were largely unchanged (Supplemental Table S4; Supplemental Figure S7); however, heterogeneity was not present anymore (Cochran's $Q$ value $=6.55, p=0.36$ ).

\section{Causal Estimates for Desaturase Activities on Risk of Coronary Artery Disease}

We found evidence for a causal total effect of D6D $(\mathrm{OR}=1.06$ [1.02-1.11]) on the risk of CAD (Table 2, Supplemental Figure S4) with no indication of heterogeneity. The MR-Egger estimates were lower and not significant anymore compared to the IVW estimates (Supplemental Table S3). For D5D, we found a positive total effect $(\mathrm{OR}=1.03$ [1.01-1.05]) on the risk of CAD (Table 2, Supplemental Figure S5) with no indication of heterogeneity. However, MR-Egger estimates were lower than the ones from the IVW method and not significant. Furthermore, the MR-Egger test indicated directional horizontal pleiotropy for D5D and CAD, pointing to a biased IVW estimate (Supplemental Table S3). When we excluded one possibly outlying SNP (rs61897792) the indication for directional horizontal pleiotropic effects for D5D and CAD disappeared (Supplemental Table S5; Supplemental Figure S8).

Sensitivity Analyses

In sensitivity analyses, we additionally included genome-wide significant instruments $\left(p<9.36 \times 10^{-9}\right)$ for D5D. For both endpoints, there was evidence for heterogeneity between the SNPs (T2DM: Cochran's Q value $=31.15, p=0.0006$; CAD: Cochran's $Q$ value $=20.46, p=0.03$ ). The indication for directional horizontal pleiotropy with regards to CAD remained (Supplemental Table S3). The observed positive associations for T2DM (OR = 1.04 [1.02-1.06]) and CAD (OR = 1.03 [1.01-1.06]) did not change (Table 2, Supplemental Figure S5). Exclusion of the previous two outlying SNPs (rs174602, rs508768) influenced the IVW effect estimate for T2DM only marginally (Supplemental Table S4; Supplemental Figures S6 and S7). Exclusion of one possibly outlying SNP (rs61897792) did not change the IVW effect estimates for CAD, but weakened the indication for directional horizontal pleiotropic effects with regards to D5D and CAD (Supplemental Table S5; Supplemental Figure S8). The CAUSE method suggested indication for causal association for both desaturases with T2DM and CAD (Supplemental Figures S9-S12). 
Table 2. Total and direct effects of estimated desaturase activities and risk of type 2 diabetes and coronary artery disease.

\begin{tabular}{|c|c|c|c|c|c|c|c|c|}
\hline & & & & T2DM & & & CAD & \\
\hline & & Method & N (SNPs) * & OR $(95 \% \mathrm{CI})$ & $p$-Value & $\mathbf{N}($ SNPs) + & OR $(95 \% \mathrm{CI})$ & $p$-Value \\
\hline \multirow[t]{5}{*}{ D6D } & \multirow[t]{3}{*}{ instruments from $F A D S$} & IVW & 6 & $\begin{array}{c}1.08 \\
(1.06-1.09)\end{array}$ & $<0.001$ & 6 & $\begin{array}{c}1.06 \\
(1.02-1.11)\end{array}$ & 0.008 \\
\hline & & MVIVW & 10 & $\begin{array}{c}1.03 \\
(0.94-1.12)\end{array}$ & 0.528 & 10 & $\begin{array}{c}1.00 \\
(0.93-1.07)\end{array}$ & 0.971 \\
\hline & & MVIVW $\ddagger$ & 10 & $\begin{array}{c}1.03 \\
(0.99-1.16)\end{array}$ & & 10 & $\begin{array}{c}1.01 \\
(0.91-1.12)\end{array}$ & \\
\hline & \multirow[t]{2}{*}{ instruments from $F A D S$ and genome-wide hits } & MVIVW & 12 & $\begin{array}{c}1.03 \\
(0.95-1.10)\end{array}$ & 0.514 & 12 & $\begin{array}{c}1.00 \\
(0.95-1.06)\end{array}$ & 0.907 \\
\hline & & MVIVW $\ddagger$ & 12 & $\begin{array}{c}1.01 \\
(0.94-1.10)\end{array}$ & & 12 & $\begin{array}{c}1.00 \\
(0.96-1.15)\end{array}$ & \\
\hline \multirow[t]{6}{*}{ D5D } & \multirow[t]{3}{*}{ instruments from $F A D S$} & IVW & 9 & $\begin{array}{c}1.03 \\
(1.01-1.04)\end{array}$ & $<0.001$ & 9 & $\begin{array}{c}1.03 \\
(1.01-1.05)\end{array}$ & 0.017 \\
\hline & & MVIVW & 10 & $\begin{array}{c}1.00 \\
(0.96-1.04)\end{array}$ & 0.824 & 10 & $\begin{array}{c}1.04 \\
(1.01-1.08)\end{array}$ & 0.021 \\
\hline & & MVIVW $\ddagger$ & 10 & $\begin{array}{c}1.02 \\
(0.98-1.04)\end{array}$ & & 10 & $\begin{array}{c}1.04 \\
(1.01-1.15)\end{array}$ & \\
\hline & \multirow[t]{3}{*}{ instruments from FADS and genome-wide hits } & IVW & 11 & $\begin{array}{c}1.04 \\
(1.02-1.06)\end{array}$ & $<0.001$ & 11 & $\begin{array}{c}1.03 \\
(1.01-1.06)\end{array}$ & 0.017 \\
\hline & & MVIVW & 12 & $\begin{array}{c}1.00 \\
(0.96-1.03)\end{array}$ & 0.845 & 12 & $\begin{array}{c}1.03 \\
(0.99-1.06)\end{array}$ & 0.108 \\
\hline & & MVIVW $\ddagger$ & 12 & $\begin{array}{c}1.02 \\
(0.99-1.05)\end{array}$ & & 12 & $\begin{array}{c}1.04 \\
(0.99-1.07)\end{array}$ & \\
\hline
\end{tabular}

CAD, coronary artery disease; CI, confidence interval; D5D, delta-5-desaturase; D6D, delta-6-desaturase; IVW, inverse variance weighted method; MVIVW, multivariable inverse variance weighted method; OR, Odds ratio; T2DM, type 2 diabetes. * Of the 11/7 SNPs associated with D5D/D6D, 11/7 were available in the GWAS of T2DM [18]. After harmonization and removal of palindromic SNPs with intermediate allele frequencies, 9/6 SNPs were included in the MR analysis on T2DM. For MVMR a combined set of 10 SNPs was used. + Of the 11/7 SNPs associated with D5D/D6D, 10/6 were available in the GWAS of CAD [19]. After harmonization and removal of palindromic SNPs with intermediate allele frequencies, 9/6 SNPs were included in the MR analysis on CAD. For MVMR a combined set of 10 SNPs was used. $\ddagger$ adjusting for weak instruments in MVMR, but not for correlation structure between instruments. 


\subsubsection{Multivariable Mendelian Randomization}

Estimates for Causal Direct Effects of Desaturase Activities on Risk of Type 2 Diabetes and Coronary Artery Disease

All independent FADS-gene-wide significant variants $(p<$ [0.05/169]) from both desaturases were combined leading to ten variants of which all were available within the outcome GWAS (Supplemental Table S6). In the multivariable MR approach, we could not precisely allocate the causal effect on T2DM to one or the other desaturase. While for D6D, we still found a small not significant direct effect on the risk of T2DM (OR = 1.03 [0.94-1.12]); for D5D, there was no direct causal effect (OR = 1.00 [0.96-1.04]). For CAD, the total causal effect of D6D was fully attenuated in multivariable MR (OR = 1.00 [0.93-1.07]) (Table 2); however, a significant direct causal effect of D5D on the risk of CAD could be observed $(\mathrm{OR}=1.04$ [1.01-1.08]) (Table 2). Though, we had indication for weak instruments with F-statistic $<10$ $(\mathrm{D} 5 \mathrm{D}=9.91, \mathrm{D} 6 \mathrm{D}=2.23)$. We repeated the multivariable MR by accounting for weak instruments and received comparable direct effect estimate for D5D (OR = 1.04 [1.01-1.15]) (Table 2).

Sensitivity Analyses

Additional inclusion of genome-wide significant instruments did not change the observation of no significant direct causal effect of D6D on the investigated outcomes while the direct effect of D5D on CAD was slightly attenuated (OR $=1.03$ [0.99-1.06]). However, the F-statistics were higher $(\mathrm{D} 5 \mathrm{D}=14.41, \mathrm{D} 6 \mathrm{D}=3.06)$. When we accounted for weak instruments, the estimates did not largely change (Table 2).

The multivariable MR-Egger regression showed intercepts that were close to zero, not indicating presence of directional horizontal pleiotropy (Supplemental Table S7). Exclusion of outlying instruments (rs174602, rs508768) did not markedly influence estimates, however, they lost precision (e.g., direct effect of D5D on CAD using only FADS-instruments: 1.03 [0.97-1.09], $p$-value $=0.336$ ) (Supplemental Table S8). Exclusion of an additional outlying instrument (rs61897792) did not further change effect estimates (Supplemental Table S9).

\subsubsection{Investigation of Confounding by Linkage Disequilibrium}

Finally, when we excluded instruments from FADS2 that are in LD with FADS1-SNPs to rule out confounding by LD (Supplemental Figure S13), we observed considerably stronger positive total effect of D6D on T2DM (1.12 [1.06-1.18]) and CAD (1.12 [1.04-1.21]) (Table 3). While the effect estimate was comparable in multivariable MR for $\mathrm{CAD}$, these analyses lost precision and no significant direct effects were observed. When we excluded one SNP (rs174602) from the FADS2 instruments that was reported to be associated with FADS1 expression [43], the results did not change (Supplemental Table S10) When we included only genome-wide significant hits outside from FADS for D5D, we still observed positive total effect on T2DM (1.04 [0.99-1.08]) and CAD (1.04 [0.98-1.11]), although not significant. Multivariable MR did not indicate direct effects of D5D on risk of T2DM or CAD (Table 3). 
Table 3. Total and direct effects of estimated desaturase activities and risk of type 2 diabetes and coronary artery disease accounting for confounding by LD.

\begin{tabular}{|c|c|c|c|c|c|c|c|c|c|}
\hline & \multirow[b]{2}{*}{ Method } & \multicolumn{3}{|c|}{ T2DM } & \multicolumn{5}{|c|}{ CAD } \\
\hline & & N (SNPs) & OR $(95 \% \mathrm{CI})$ & $p$-Value & $\begin{array}{c}\text { Intercept (SE), } \\
p \text {-Value }\end{array}$ & N (SNPs) & OR $(95 \% \mathrm{CI})$ & $p$-Value & $\begin{array}{c}\text { Intercept (SE), } \\
p \text {-Value }\end{array}$ \\
\hline \multirow[t]{3}{*}{ D6D } & MR-Egger & $3+$ & $\begin{array}{c}0.87 \\
(0.55-1.37)\end{array}$ & 0.538 & $0.050(0.045), 0.267$ & $3+$ & $\begin{array}{c}1.26 \\
(0.21-7.70)\end{array}$ & 0.804 & $-0.022(0.176), 0.902$ \\
\hline & IVW * & $3+$ & $\begin{array}{c}1.12 \\
(1.06-1.18)\end{array}$ & $<0.001$ & & $3+$ & $\begin{array}{c}1.12 \\
(1.04-1.21)\end{array}$ & 0.002 & \\
\hline & MVIVW * & $3+$ & $\begin{array}{c}0.74 \\
(0.34-1.62)\end{array}$ & 0.453 & & $3+$ & $\begin{array}{c}1.12 \\
(0.34-3.69)\end{array}$ & 0.850 & \\
\hline \multirow[t]{3}{*}{ D5D } & $\mathrm{IVW}^{*}$ & $2 \ddagger$ & $\begin{array}{c}1.04 \\
(0.99-1.08)\end{array}$ & 0.087 & & $2 \ddagger$ & $\begin{array}{c}1.04 \\
(0.98-1.11)\end{array}$ & 0.236 & \\
\hline & MVIVW * & $2 \ddagger$ & $\begin{array}{c}1.01 \\
(0.95-1.07)\end{array}$ & 0.790 & & $2 \ddagger$ & $\begin{array}{c}1.00 \\
(0.93-1.08)\end{array}$ & 0.996 & \\
\hline & MVIVW * & $3+$ & $\begin{array}{c}1.29 \\
(0.75-2.21)\end{array}$ & 0.362 & & $3+$ & $\begin{array}{c}1.00 \\
(0.48-2.09)\end{array}$ & 1.000 & \\
\hline
\end{tabular}

* Fixed effect model; † including only FADS1-independent $\left(\mathrm{R}^{2}<0.45\right)$ FADS2 variants (rs174602, rs498793, rs7118175); † including only genome-wide hits for D5D (rs2608073, rs11644601). 


\section{Discussion}

Within this study, we applied a two-sample MR approach to investigate causal effects of estimated desaturase activities on the risk of T2DM and CAD. Our univariable MR indicated that PUFA-generating desaturases are causally involved in the development of both, T2DM and CAD. For D6D these effects became more prominent if we accounted for confounding by LD in our genetic instruments. However, due to low precision, attribution of the direct causal effect on T2DM to D6D activity remains suggestive. For CAD, results from the multivariable MR suggested a direct effect only for D5D activity, but we were not able to exclude confounding by LD. It was therefore not possible to disentangle the causal impact of the single desaturases on CAD risk.

\subsection{D6D and Risk of Type 2 Diabetes and Coronary Artery Disease}

The D6D catalyzes the desaturation of linoleic acid (LA) that shows inverse association with cardiovascular disease mortality [44] and cardiovascular events [45]. The product of the D6D, $\gamma$-linolenic acid (GLA) and higher D6D activity itself are positively associated with T2DM [2,14]. Our results from univariable MR are in line with a previous MR study reporting that a genetically determined high D6D activity [14] predicted higher T2DM risk. Also, low levels of LA, representing a D6D substrate, were associated with increased risk of T2DM in recent MR analysis [12]. However, selecting instruments for D6D is affected by the strong LD in the FADS gene cluster, where one LD block spans FADS1 and parts of the FADS2 gene [1]. Therefore, genetic instruments for D6D (from FADS2) cannot be easily separated from those for D5D (FADS1).

We tried to overcome this problem of confounding by LD for D6D by restricting the selected instruments to those from the FADS2 gene that are not in LD with variants from FADS1. This sensitivity analysis revealed stronger total effects of D6D on both T2DM and CAD, which further supports a causal role of D6D. Still, due to the considerable loss of precision in the multivariable MR the allocation of the T2DM-risk augmenting effect of D6D activity exclusively to those PUFAs being substrate or product of D6D activity (LA and GLA) remains relatively uncertain. More likely, downstream formation of PUFAs from GLA mediate an important part of the total effect of D6D.

\subsection{D5D and Risk of Type 2 Diabetes and Coronary Artery Disease}

Regarding D5D, alignment of our results from univariable MR with non-genetic observational studies is more complex. D5D, catalyzes the conversion of DGLA to arachidonic acid (AA). Our univariable MR suggested links of higher D5D with higher risk of T2DM. This is in line with a recent MR study showing higher T2DM risk with higher levels of AA [12]. In contrast to that, observational studies relate higher estimated D5D activity and higher AA levels to lower T2DM risk [2,14]. A direct link of D5D with T2DM was, however, not observable in multivariable MR. Also, instruments for D5D (FADS1) are largely confounded by LD with FADS2 variants and we were not able to overcome this LD problem by using instruments outside the FADS region. Therefore, our finding of a higher T2DM risk among participants with genetically high D5D activity in univariable MR reflects most likely the genetic linkage with D6D rather than an independent effect.

In contrast to T2DM, the observational evidence that links higher estimated D5D activity to lower cardiovascular mortality and CHD risk is rather limited [4,5], where an inverse association of high D5D activity was limited to participants who were homozygous for the major allele (AA genotype; rs174547) [5]. A previous MR on fatty acids showed a positive effect of higher AA levels on CVD risk [13], although this study did not evaluate fatty acid ratios as estimate of D5D activity. While our univariable MR analysis supports a total causal effect of D5D activity on CAD, interpreting these findings as reliable evidence for causal effects is limited. We had significant MR-Eggers test, indicating that directional (unbalanced) horizontal pleiotropy was present or that the InSIDE assumption was violated or both [46]. We did not identify outliers, that could explain this finding. Furthermore, although we identified a significant direct effect of D5D on CAD when using only FADS-instruments, 
this result was sensitive to inclusion of genome-wide instruments and outlier exclusions. As pointed out above, it is virtually impossible to account for confounding by LD. Restricting our analysis to variants outside of the FADS-gene region hampered precision of the D5D estimates to an extent that made them uninformative. Hence, we do not consider effect of D5D on CAD risk to be robust and will therefore restrain from causal interpretation.

\subsection{Biological Mechanisms}

PUFAs play a crucial role in cell membrane fluidity and thereby influence insulin receptor binding affinity and endothelial function [47] which might represent one mechanism how altered desaturation of fatty acids influences the risks of T2DM [48] and CAD [49]. Additionally, PUFAs affect transcription factors such as sterol regulatory element binding protein 1 and peroxisome proliferators activating receptors regulating genes involved in control of lipid flux into and out of the liver, which is important in terms of reducing hepatic lipid accumulation and hence hepatic insulin resistance [50]. Furthermore, PUFAs are substrate for the formation of various lipid-related metabolites, e.g., eicosanoids, leukotriens, prostaglandins, thromboxanes, lipoxins, endo-cannabinoids, or resolvins, which themselves are highly bioactive [47]. AA-related eicosanoids may increase vasoconstriction and platelet activation and aggregation, promoting atherosclerotic plaque formation as pathological processes in the development of CAD [51].

\subsection{Strengths and Limitations}

By using instruments from the FADS region, we used well established genetic candidates from the genic region of the investigated exposures that explain up to $\sim 30 \%$ (rs174555 and D5D) of the variance in the traits ( $\mathrm{R}^{2}$ ranging from 5 to $10 \%$ for $\mathrm{D} 6 \mathrm{D}$ and 1 to $30 \%$ for D5D). Although we had indication for weak instruments within the multivariable $\mathrm{MR}$, we retrieved comparable estimates when we accounted for that. Besides genetic candidates, we also used genome-wide screen and identified two novel hits for D5D that have not been reported by previous GWAS on estimated desaturase activities [8,9]. Although our genome-wide significance cut-off might be too strict, we would not have found additional hits using the common threshold of $p<5 \times 10^{-8}$. Furthermore, we wanted to be more conservative, as we did not replicate those findings in an independent population. Nevertheless, our MR results incorporating those genome-wide hits were comparable to the main analysis when using only FADS variants.

We minimized potential incorrect causal inference due to horizontal pleiotropy by restricting genetic instruments on those that plausibly act directly on the traits as they were located in the coding genes [52]. Furthermore, we corrected for correlated and uncorrelated horizontal pleiotropy using CAUSE method and still retained indication for causal effect of both desaturases and cardiometabolic outcomes. Additionally, there must be no LD with other variants that might influence the expression or activity of a different protein as this can reintroduce confounding by LD [52]. Similar to the pleiotropy situation, this would violate key assumptions of MR [50]. We addressed confounding by LD by accounting for the LD structure in the main analysis and by restricting genetic instruments to those that are not in LD with instruments for the respective other desaturase. Still, there might be criticism as the two instruments (rs498793, rs7118175) for D6D used in sensitivity analyses showed significant associations with D5D levels and might therefore be considered as not independent from FADS1; however, we did not identify genome-wide significant instruments for D6D outside of FADS which could have been used instead.

One limitation of our analysis lies in the exclusion of prevalent cases in the EPIC-Potsdam sample which was used for instrument selection and might have weakened the exposure betas. However, compared to the overall study sample this number was small and we aimed to exclude bias from reverse confounding due to the fact that prevalent cases might have different desaturase activity [52]. Also, our multivariable MR might be underpowered; however, we did no power calculation, as this is_-to our knowledge-only developed for continuous outcomes [53]. Furthermore, we did not directly 
measure desaturase activities. In humans, D6D and D5D are mainly expressed in the liver [54,55]. However, direct measurement of liver desaturase activity would require liver biopsies which is not possible in population-based settings. Therefore, product-to-precursor ratios of fatty acids measured in blood fractions represent well-established surrogate markers of estimated liver desaturase activity in epidemiological research [1,56]. Another limitation refers to the selection of the outcome GWAS for CAD. Ideally, the exposure and outcome data should arise from the same population in terms of ancestry. For T2DM, this was fulfilled; however, for CAD, also Asian individuals contributed to the meta-analysis [19]. Nevertheless, this should not increase the likelihood of finding an association when there is none [57], hence our results on D6D and CAD might still be informative in terms of effect directions of causality [31].

\section{Conclusions}

In conclusion, our results suggest that D6D is causally linked to cardiometabolic risk. However, the effect is likely not directly due to its substrate or product fatty acids, but rather due to the downstream production of fatty acids (DGLA and AA) and their products resulting from high D6D activity. For D5D, our MR approach suggests a causal risk-increasing effect for T2DM and CAD risk; however, we were not able to fully rule out confounding by LD. Interventions that affect desaturase activities in PUFA metabolism may have an impact on the pathogenesis of cardiometabolic diseases.

Supplementary Materials: The following are available online at http://www.mdpi.com/2072-6643/12/8/2261/s1, Supplemental Table S1 Instruments from FADS gene cluster for D6D $\left(\mathrm{R}^{2}<0.3\right)$ and their association with T2DM or CAD. Supplemental Table S2 Instruments from FADS gene cluster for D5D $\left(\mathrm{R}^{2}<0.3\right)$ and genome-wide hits and their association with T2DM or CAD. Supplemental Table S3 Total effects of estimated desaturase activities and risk of type 2 diabetes or coronary artery disease using MR-Egger. Supplemental Table S4 Total effects of estimated delta-5-desaturase activity and risk of type 2 diabetes excluding outlying variants. Supplemental Table S5 Total effects of estimated delta-5-desaturase activity and risk of coronary artery disease excluding outlying variant. Supplemental Table S6 Association between genetic instruments used in multivariable MR and estimated desaturase activities and risk of type 2 diabetes or coronary artery disease. Supplemental Table S7 Direct effects of estimated desaturase activities and risk of type 2 diabetes or coronary artery disease using multivariable MR-Egger. Supplemental Table S8 Direct effects of estimated desaturase activities and risk of type 2 diabetes or coronary artery disease excluding outlying variants. Supplemental Table S9 Direct effects of estimated desaturase activities and risk of type 2 diabetes or coronary artery disease excluding outlying variants. Supplemental Table S10 Total and direct effects of estimated desaturase activities and risk of type 2 diabetes and coronary artery disease accounting for confounding by LD, excluding rs174602. Supplemental Figure S1 Flow-chart of final study population. Supplemental Figure S2 Manhattan plots visualizing GWAS results for D6D and D5D. Supplemental Figure S3 LD-Plot visualizing the degree of linkage disequilibrium between genetic instruments from FADS gene region evaluated within the study. Supplemental Figure S4 Scatter plots visualizing genetic associations with D6D and type 2 diabetes and coronary artery disease. Supplemental Figure S5 Scatter plots visualizing genetic associations with D5D and type 2 diabetes and coronary artery disease. Supplemental Figure S6 Radial MR plot for D5D and type 2 diabetes. Supplemental Figure S7 Scatter plots visualizing genetic associations with D5D and type 2 diabetes after outlier removal. Supplemental Figure S8 Scatter plots visualizing genetic associations with D5D and coronary artery disease after outlier removal. Supplemental Figure S9 Effect-size estimates and variant-level contribution to CAUSE test statistics for D6D and type 2 diabetes. Supplemental Figure S10 Effect-size estimates and variant-level contribution to CAUSE test statistics for D6D and coronary artery disease. Supplemental Figure S11 Effect-size estimates and variant-level contribution to CAUSE test statistics for D5D and type 2 diabetes. Supplemental Figure S12 Effect-size estimates and variant-level contribution to CAUSE test statistics for D5D and coronary artery disease. Supplemental Figure S13 LD-Plot visualizing the degree of linkage disequilibrium between 143 genetic instruments for D6D from FADS gene region

Author Contributions: S.J., C.W. and M.B.S. conceptualized the project and designed the analysis plan. S.J. analyzed the data. P.H. acquired data. S.J. and R.C. performed quality control of genetic data. S.J., C.W., R.C. and M.B.S. interpreted the data. S.J. wrote the first draft of the manuscript. C.W., R.C., P.H. and M.B.S. reviewed the manuscript. Both S.J. and M.B.S. had access to all data for this study and take responsibility for the manuscript contents. All authors have read and agreed to the published version of the manuscript.

Funding: The recruitment phase of the EPIC-Potsdam Study was supported by the Federal Ministry of Science, Germany (01 EA 9401) and the European Union (SOC 95201408 05F02). The follow-up of the EPIC-Potsdam Study was supported by German Cancer Aid (70-2488-Ha I) and the European Community (SOC 98200769 05F02). This work was furthermore supported by a grant from the German Ministry of Education and Research (BMBF) and the State of Brandenburg (DZD grant 82DZD00302). 
Acknowledgments: We thank the Human Study Centre (HSC) of the German Institute of Human Nutrition Potsdam-Rehbrücke, namely the trustee and the data hub for the processing, and the participants for the provision of the data, the biobank for the processing of the biological samples and the head of the HSC, Manuela Bergmann, for the contribution to the study design and leading the underlying processes of data generation. The authors are indebted to Hans Cremers for the excellent performance of the fatty acid analysis. Summary-level data for genetic associations with the cardiovascular disease and type 2 diabetes outcomes have been contributed by the CARDIoGRAMplusC4D (http://www.cardiogramplusc4d.org/data-downloads/; accessed 12.08.2019) and DIAGRAM consortia (http://diagram-consortium.org/downloads.html; accessed 08.08.2019). The authors thank all investigators for sharing these data.

Conflicts of Interest: The authors declare no conflict of interest.

Ethics Approval and Consent to Participate: All participants provided written informed consent. The EPICPotsdam study was approved by the ethics committee of the State of Brandenburg, Germany. All procedures were in accordance with the ethical standards of the institutional and/or national research committee and with the 1964 Helsinki declaration and its later amendments or comparable ethical standards.

Availability of Data and Materials: The EPIC-Potsdam datasets analyzed during the current study are not publicly available due to data protection regulations. In accordance with German Federal and State data protection regulations, epidemiological data analyses of EPIC-Potsdam may be initiated upon an informal inquiry addressed to the secretariate of the Human Study Center (Office.HSZ@dife.de). Each request will then have to pass a formal process of application and review by the respective PI and a scientific board. Summary-level data for genetic associations with the cardiovascular disease and type 2 diabetes outcomes have been contributed by the CARDIoGRAMplusC4D (http://www.cardiogramplusc4d.org/data-downloads/; accessed on 12 August 2019) and DIAGRAM consortia (http://diagram-consortium.org/downloads.html; accessed on 8 August 2019). The authors thank all investigators for sharing these data.

Code Availability: Software codes are available upon request.

\section{References}

1. Kröger, J.; Schulze, M.B. Recent insights into the relation of Delta5 desaturase and Delta6 desaturase activity to the development of type 2 diabetes. Curr. Opin. Lipidol. 2012, 23, 4-10. [CrossRef]

2. Forouhi, N.G.; Imamura, F.; Sharp, S.J.; Koulman, A.; Schulze, M.B.; Zheng, J.; Ye, Z.; Sluijs, I.; Guevara, M.; Huerta, J.M.; et al. Association of plasma phospholipid n-3 and n-6 polyunsaturated fatty acids with type 2 diabetes: The EPIC-interAct case-cohort study. PLoS Med. 2016, 13, e1002094. [CrossRef] [PubMed]

3. Wu, J.H.Y.; Marklund, M.; Imamura, F.; Tintle, N.; Ardisson Korat, A.V.; de Goede, J.; Zhou, X.; Yang, W.S.; de Oliveira Otto, M.C.; Kroger, J.; et al. Omega-6 fatty acid biomarkers and incident type 2 diabetes: Pooled analysis of individual-level data for 39740 adults from 20 prospective cohort studies. Lancet Diabetes Endocrinol. 2017, 5, 965-974. [CrossRef]

4. Warensjo, E.; Sundstrom, J.; Vessby, B.; Cederholm, T.; Riserus, U. Markers of dietary fat quality and fatty acid desaturation as predictors of total and cardiovascular mortality: A population-based prospective study. Am. J. Clin. Nutr. 2008, 88, 203-209. [CrossRef] [PubMed]

5. Lu, Y.; Vaarhorst, A.; Merry, A.H.; Dolle, M.E.; Hovenier, R.; Imholz, S.; Schouten, L.J.; Heijmans, B.T.; Muller, M.; Slagboom, P.E.; et al. Markers of endogenous desaturase activity and risk of coronary heart disease in the CAREMA cohort study. PLoS ONE 2012, 7, e41681. [CrossRef]

6. Davey Smith, G.; Ebrahim, S. 'Mendelian randomization': Can genetic epidemiology contribute to understanding environmental determinants of disease? Int. J. Epidemiol. 2003, 32, 1-22. [CrossRef] [PubMed]

7. Marquardt, A.; Stohr, H.; White, K.; Weber, B.H. cDNA cloning, genomic structure, and chromosomal localization of three members of the human fatty acid desaturase family. Genomics 2000, 66, 175-183. [CrossRef]

8. De Toro-Martin, J.; Guenard, F.; Rudkowska, I.; Lemieux, S.; Couture, P.; Vohl, M.C. A common variant in ARHGEF10 alters delta- 6 desaturase activity and influence susceptibility to hypertriglyceridemia. J. Clin. Lipidol. 2018, 12, 311-320. [CrossRef]

9. Marklund, M.; Morris, A.P.; Mahajan, A.; Ingelsson, E.; Lindgren, C.M.; Lind, L.; Riserus, U. Genome-wide association studies of estimated fatty acid desaturase activity in serum and adipose tissue in elderly individuals: Associations with insulin sensitivity. Nutrients 2018, 10, 1791. [CrossRef]

10. O'Neill, C.M.; Minihane, A.M. The impact of fatty acid desaturase genotype on fatty acid status and cardiovascular health in adults. Proc. Nutr. Soc. 2017, 76, 64-75. [CrossRef] 
11. Brayner, B.; Kaur, G.; Keske, M.A.; Livingstone, K.M. FADS polymorphism, omega-3 fatty acids and diabetes risk: A systematic review. Nutrients 2018, 10, 758. [CrossRef] [PubMed]

12. Yuan, S.; Larsson, S.C. Association of genetic variants related to plasma fatty acids with type 2 diabetes mellitus and glycaemic traits: A Mendelian randomisation study. Diabetologia 2020, 63, 116-123. [CrossRef]

13. Yuan, S.; Back, M.; Bruzelius, M.; Mason, A.M.; Burgess, S.; Larsson, S. FADS polymorphism, omega-3 fatty acids and diabetes risk: A systematic review. Nutrients 2019, 11, 3001. [CrossRef] [PubMed]

14. Kröger, J.; Zietemann, V.; Enzenbach, C.; Weikert, C.; Jansen, E.H.; Doring, F.; Joost, H.G.; Boeing, H.; Schulze, M.B. Erythrocyte membrane phospholipid fatty acids, desaturase activity, and dietary fatty acids in relation to risk of type 2 diabetes in the European Prospective Investigation into Cancer and Nutrition (EPIC)-Potsdam Study. Am. J. Clin. Nutr. 2011, 93, 127-142. [CrossRef]

15. Burgess, S.; Thompson, S.G. Multivariable Mendelian randomization: The use of pleiotropic genetic variants to estimate causal effects. Am. J. Epidemiol. 2015, 181, 251-260. [CrossRef] [PubMed]

16. Boeing, H.; Wahrendorf, J.; Becker, N. EPIC-Germany-A source for studies into diet and risk of chronic diseases. European Investigation into Cancer and Nutrition. Ann. Nutr. Metab. 1999, 43, 195-204. [CrossRef] [PubMed]

17. Stefan, N.; Fritsche, A.; Weikert, C.; Boeing, H.; Joost, H.G.; Haring, H.U.; Schulze, M.B. Plasma fetuin-A levels and the risk of type 2 diabetes. Diabetes 2008, 57, 2762-2767. [CrossRef] [PubMed]

18. Mahajan, A.; Taliun, D.; Thurner, M.; Robertson, N.R.; Torres, J.M.; Rayner, N.W.; Payne, A.J.; Steinthorsdottir, V.; Scott, R.A.; Grarup, N.; et al. Fine-mapping type 2 diabetes loci to single-variant resolution using high-density imputation and islet-specific epigenome maps. Nat. Genet. 2018, 50, 1505-1513. [CrossRef]

19. Nelson, C.P.; Goel, A.; Butterworth, A.S.; Kanoni, S.; Webb, T.R.; Marouli, E.; Zeng, L.; Ntalla, I.; Lai, F.Y.; Hopewell, J.C.; et al. Association analyses based on false discovery rate implicate new loci for coronary artery disease. Nat. Genet. 2017, 49, 1385-1391. [CrossRef]

20. Langenberg, C.; Sharp, S.J.; Franks, P.W.; Scott, R.A.; Deloukas, P.; Forouhi, N.G.; Froguel, P.; Groop, L.C.; Hansen, T.; Palla, L.; et al. Gene-lifestyle interaction and type 2 diabetes: The EPIC interact case-cohort study. PLoS Med. 2014, 11, e1001647. [CrossRef]

21. Goldstein, J.I.; Crenshaw, A.; Carey, J.; Grant, G.B.; Maguire, J.; Fromer, M.; O’Dushlaine, C.; Moran, J.L.; Chambert, K.; Stevens, C.; et al. zCall: A rare variant caller for array-based genotyping: Genetics and population analysis. Bioinformatics 2012, 28, 2543-2545. [CrossRef] [PubMed]

22. Grove, M.L.; Yu, B.; Cochran, B.J.; Haritunians, T.; Bis, J.C.; Taylor, K.D.; Hansen, M.; Borecki, I.B.; Cupples, L.A.; Fornage, M.; et al. Best practices and joint calling of the HumanExome BeadChip: The CHARGE Consortium. PLoS ONE 2013, 8, e68095. [CrossRef]

23. Anderson, C.A.; Pettersson, F.H.; Clarke, G.M.; Cardon, L.R.; Morris, A.P.; Zondervan, K.T. Data quality control in genetic case-control association studies. Nat. Protoc. 2010, 5, 1564-1573. [CrossRef] [PubMed]

24. Guo, Y.; He, J.; Zhao, S.; Wu, H.; Zhong, X.; Sheng, Q.; Samuels, D.C.; Shyr, Y.; Long, J. Illumina human exome genotyping array clustering and quality control. Nat. Protoc. 2014, 9, 2643-2662. [CrossRef]

25. Das, S.; Forer, L.; Schonherr, S.; Sidore, C.; Locke, A.E.; Kwong, A.; Vrieze, S.I.; Chew, E.Y.; Levy, S.; McGue, M.; et al. Next-generation genotype imputation service and methods. Nat. Genet. 2016, 48, 1284-1287. [CrossRef] [PubMed]

26. McCarthy, S.; Das, S.; Kretzschmar, W.; Delaneau, O.; Wood, A.R.; Teumer, A.; Kang, H.M.; Fuchsberger, C.; Danecek, P.; Sharp, K.; et al. A reference panel of 64,976 haplotypes for genotype imputation. Nat. Genet. 2016, 48, 1279-1283.

27. Loh, P.R.; Palamara, P.F.; Price, A.L. Fast and accurate long-range phasing in a UK Biobank cohort. Nat. Genet. 2016, 48, 811-816. [CrossRef]

28. Li, H. A statistical framework for SNP calling, mutation discovery, association mapping and population genetical parameter estimation from sequencing data. Bioinformatics 2011, 27, 2987-2993. [CrossRef]

29. Rayner, N.W.; Robertson, N.; Mahajan, A.; McCarthy, M.I. A Suite of Programs for Pre- and Postimputation Data Checking. Available online: www.well.ox.ac.uk/ wrayner/tools (accessed on 15 April 2019).

30. Marchini, J.; Howie, B.; Myers, S.; McVean, G.; Donnelly, P. A new multipoint method for genome-wide association studies by imputation of genotypes. Nat. Genet. 2007, 39, 906-913. [CrossRef] 
31. Hemani, G.; Zheng, J.; Elsworth, B.; Wade, K.H.; Haberland, V.; Baird, D.; Laurin, C.; Burgess, S.; Bowden, J.; Langdon, R.; et al. The MR-Base platform supports systematic causal inference across the human phenome. Elife 2018, 7, e34408. [CrossRef]

32. Yavorska, O.O.; Burgess, S. MendelianRandomization: An R package for performing Mendelian randomization analyses using summarized data. Int. J. Epidemiol. 2017, 46, 1734-1739. [CrossRef] [PubMed]

33. Bowden, J.; Spiller, W.; Del Greco, M.F.; Sheehan, N.; Thompson, J.; Minelli, C.; Davey Smith, G. Improving the visualization, interpretation and analysis of two-sample summary data Mendelian randomization via the Radial plot and Radial regression. Int. J. Epidemiol. 2018, 47, 1264-1278. [CrossRef] [PubMed]

34. Sanderson, E.; Spiller, W.; Bowden, J. Testing and correcting for weak and pleiotropic instruments in two-sample multivariable mendelian randomisation. BioRxiv 2020. Available online: https://www.biorxiv. org/content/10.1101/2020.04.02.021980v1 (accessed on 3 April 2020). [CrossRef]

35. Purcell, S.; Neale, B.; Todd-Brown, K.; Thomas, L.; Ferreira, M.A.; Bender, D.; Maller, J.; Sklar, P.; de Bakker, P.I.; Daly, M.J.; et al. PLINK: A tool set for whole-genome association and population-based linkage analyses. Am. J. Hum. Genet. 2007, 81, 559-575. [CrossRef]

36. Barrett, J.C.; Fry, B.; Maller, J.; Daly, M.J. Haploview: Analysis and visualization of LD and haplotype maps. Bioinformatics 2005, 21, 263-265. [CrossRef]

37. Cunningham, F.; Amode, M.R.; Barrell, D.; Beal, K.; Billis, K.; Brent, S.; Carvalho-Silva, D.; Clapham, P.; Coates, G.; Fitzgerald, S.; et al. Ensembl 2015. Nucleic Acids Res. 2015, 43, D662-D669. [CrossRef]

38. McLaren, W.; Gil, L.; Hunt, S.E.; Riat, H.S.; Ritchie, G.R.; Thormann, A.; Flicek, P.; Cunningham, F. The ensembl variant effect predictor. Genome Biol. 2016, 17, 122. [CrossRef]

39. Burgess, S.; Dudbridge, F.; Thompson, S.G. Combining information on multiple instrumental variables in Mendelian randomization: Comparison of allele score and summarized data methods. Stat. Med. 2016, 35, 1880-1906. [CrossRef]

40. Bowden, J.; Davey Smith, G.; Burgess, S. Mendelian randomization with invalid instruments: Effect estimation and bias detection through Egger regression. Int. J. Epidemiol. 2015, 44, 512-525. [CrossRef]

41. Rees, J.M.B.; Wood, A.M.; Burgess, S. Extending the MR-Egger method for multivariable Mendelian randomization to correct for both measured and unmeasured pleiotropy. Stat. Med. 2017, 36, 4705-4718. [CrossRef]

42. Morrison, J.; Knoblauch, N.; Marcus, J.H.; Stephens, M.; He, X. Mendelian randomization accounting for correlated and uncorrelated pleiotropic effects using genome-wide summary statistics. Nat. Genet. 2020, 52, 740-747. [CrossRef] [PubMed]

43. GTEx Consortium. The Genotype-Tissue Expression (GTEx) project. Nat. Genet. 2013, 45, 580-585. [CrossRef] [PubMed]

44. Li, J.; Guasch-Ferre, M.; Li, Y.; Hu, F.B. Dietary intake and biomarkers of linoleic acid and mortality: Systematic review and meta-analysis of prospective cohort studies. Am. J. Clin. Nutr. 2020, 112, 150-167. [CrossRef] [PubMed]

45. Marklund, M.; Wu, J.H.Y.; Imamura, F.; Del Gobbo, L.C.; Fretts, A.; de Goede, J.; Shi, P.; Tintle, N.; Wennberg, M.; Aslibekyan, S.; et al. Biomarkers of dietary omega- 6 fatty acids and incident cardiovascular disease and mortality: An individual-level pooled analysis of 30 cohort studies. Circulation 2019, 139, 2422-2436. [CrossRef]

46. Burgess, S.; Thompson, S.G. Interpreting findings from Mendelian randomization using the MR-Egger method. Eur. J. Epidemiol. 2017, 32, 377-389. [CrossRef]

47. Das, U.N. A defect in Delta6 and Delta5 desaturases may be a factor in the initiation and progression of insulin resistance, the metabolic syndrome and ischemic heart disease in South Asians. Lipids Health Dis. 2010, 9, 130. [CrossRef] [PubMed]

48. Kröger, J.; Jacobs, S.; Jansen, E.H.; Fritsche, A.; Boeing, H.; Schulze, M.B. Erythrocyte membrane fatty acid fluidity and risk of type 2 diabetes in the EPIC-Potsdam study. Diabetologia 2015, 58, 282-289. [CrossRef] [PubMed]

49. Wu, H.; Ding, E.L.; Toledo, E.T.; Campos, H.; Baylin, A.; Hu, F.B.; Sun, Q. A novel fatty acid lipophilic index and risk of CHD in US men: The health professionals follow-up study. Br. J. Nutr. 2013, 110, 466-474. [CrossRef] [PubMed] 
50. Fernandez, M.L.; West, K.L. Mechanisms by which dietary fatty acids modulate plasma lipids. J. Nutr. 2005, 135, 2075-2078. [CrossRef]

51. Gleim, S.; Stitham, J.; Tang, W.H.; Martin, K.A.; Hwa, J. An eicosanoid-centric view of atherothrombotic risk factors. Cell. Mol. Life Sci. 2012, 69, 3361-3380. [CrossRef]

52. Davey Smith, G.; Hemani, G. Mendelian randomization: Genetic anchors for causal inference in epidemiological studies. Hum. Mol. Genet. 2014, 23, R89-R98. [CrossRef] [PubMed]

53. Deng, L.; Zhang, H.; Yu, K. Power calculation for the general two-sample Mendelian randomization analysis. Genet. Epidemiol. 2020, 44, 290-299. [CrossRef] [PubMed]

54. Cho, H.P.; Nakamura, M.; Clarke, S.D. Cloning, expression, and fatty acid regulation of the human delta-5 desaturase. J. Biol. Chem. 1999, 274, 37335-37339. [CrossRef]

55. Cho, H.P.; Nakamura, M.T.; Clarke, S.D. Cloning, expression, and nutritional regulation of the mammalian Delta-6 desaturase. J. Biol. Chem. 1999, 274, 471-477. [CrossRef]

56. Vaittinen, M.; Mannisto, V.; Kakela, P.; Agren, J.; Tiainen, M.; Schwab, U.; Pihlajamaki, J. Interorgan cross talk between fatty acid metabolism, tissue inflammation, and FADS2 genotype in humans with obesity. Obesity 2017, 25, 545-552. [CrossRef] [PubMed]

57. Burgess, S.; Butterworth, A.S.; Thompson, J.R. Beyond Mendelian randomization: How to interpret evidence of shared genetic predictors. J. Clin. Epidemiol. 2016, 69, 208-216. [CrossRef]

(C) 2020 by the authors. Licensee MDPI, Basel, Switzerland. This article is an open access article distributed under the terms and conditions of the Creative Commons Attribution (CC BY) license (http://creativecommons.org/licenses/by/4.0/). 